\title{
Clinical SYNTAX Score - a good predictor for renal artery stenosis in acute myocardial infarction patients: analysis from the REN-ACS trial
}

\author{
Alexandru Burlacu ${ }^{1}$, Dimitrie Siriopol' ${ }^{2}$, Ionut Nistor ${ }^{2}$, Luminita Voroneanu², Igor Nedelciuc ${ }^{1}$, \\ Cristian Statescü ${ }^{3}$ Adrian Covic ${ }^{2}$
}

\begin{abstract}
1Department of Interventional Cardiology, Cardiovascular Diseases Institute, Iasi, Romania

${ }^{2}$ Department of Nephrology, University of Medicine 'Gr. T. Popa', Iasi, Romania ${ }^{3}$ Department of Cardiology, Cardiovascular Diseases Institute, Iasi, Romania
\end{abstract}

Submitted: 31 March 2016

Accepted: 29 April 2016

Arch Med Sci 2017; 13, 4: 837-844

DOI: https://doi.org/10.5114/aoms.2016.60374

Copyright (c) 2016 Termedia \& Banach

\section{Abstract}

Introduction: In ST-elevation myocardial infarction (STEMI) patients, multisite artery disease represents a serious issue influencing evolution, outcomes and prognosis. We evaluated for the first time the power of the Myocardial Infarction SYNTAX Score (MI SS) and Clinical SYNTAX Score (MI CSS) as predictors for renal artery stenosis (RAS) in STEMI. We also stratified the study population according to the two scores, and identified the variables correlated with the higher score.

Material and methods: We used data from the REN-ACS study, which included 181 consecutive patients prospectively investigated for presence of RAS (through renal angiography), arterial stiffness (carotid-femoral pulse wave velocity, cf-PWV) and hydration status (bioimpedance). MI SS and CSS were computed.

Results: Multivariate regressions indicated that the independent variables correlated with MI SS were left ventricular ejection fraction < $40 \%$, significant RAS ( $>50 \%$, defined as RAS+), history of heart failure, and multivascular coronary disease (CAD, $p<0.03$ for each), while those correlated with MI CSS were RAS+, Cf-PWV, history of CAD, multivascular CAD, cholesterol, and total body water ( $p<0.02$ for each). In order to evaluate the ability to predict RAS + we generated receiver operating characteristics and areas under curves, and the Youden index for MI SS and CSS.

Conclusions: Both scores correlated with extensive atherosclerotic disease and presence of RAS+. A lower CSS proved to be a good predictor for exclusion of RAS+, with high specificity ( $85 \%$ ) and negative predictive value $(92 \%)$, and fair sensitivity (60\%). We aim to further pursue this line of research and design a better predictor for RAS, with the inclusion of a novel biomarker in order to increase sensitivity.

Key words: renal artery stenosis, primary percutaneous coronary intervention, ST-elevation myocardial infarction, SYNTAX Score, Clinical SYNTAX Score, predictor tool.

\section{Introduction}

In the last three decades, complex atherosclerotic coronary artery disease (CAD) and its increasing diagnostic and therapeutic arsenal gen-

\author{
Corresponding author: \\ Alexandru Burlacu MD \\ Department of Interventional \\ Cardiology \\ Cardiovascular Diseases \\ Institute \\ Bd. Carol 1, No 50 \\ 700503 Iasi, Romania \\ Phone: 0040744488580 \\ E-mail: alburlacu@yahoo.com
}


erated stirring discussions and frequent updates in risk stratification and treatment guidelines. Introduced in 2005, the SYNTAX (Synergy Between Percutaneous Coronary Intervention With Taxus and Cardiac Surgery) score (SS) is a valuable tool in therapeutic decision algorithms for multivessel CAD because it facilitates the choice between percutaneous coronary interventions ( $\mathrm{PCl}$ ) or coronary arterial bypass grafting (CABG) [1]. Subsequent studies ascertained distinctly its importance in short- and long-term prognosis assessment (major adverse cardiac events - MACE, target vessel revascularization - TVR) in this particular group of patients [2, 3]. Later research led to the development of four directions in the use of SS:

1. Elaboration and computation of derived Syntax scores (Clinical Syntax Score - CSS, Functional Syntax Score, Global Risk Classification, Residual Syntax, and CABG Syntax), through the addition of several clinical and biochemical variables to the coronary burden score computing system in order to improve its discriminatory power (for better risk stratification, higher discrimination between lower and intermediate risk patients and to improve the ability to predict MACE, stent thrombosis, or combined ischemic endpoints [4]).

2. Testing SS (and derived scores) on real-world patients (including 1- and 2-vessel disease) and proving its benefits in prognostic estimation and choice of treatment [5].

3. Inclusion of patients with acute coronary syndrome (ACS) (unstable angina/non-ST elevation myocardial infarction (non-STEMI) or ST elevation myocardial infarction (STEMI) patients) and validation of SS in the acute setting (SS becoming a good predictor for post-procedural outcomes [6]).

4. Correlation of SS with "softer" end-points (estimated glomerular filtration rate (eGFR) in stable CAD [7] and in ACS [8], high-sensitivity C-reactive protein (CRP) in ACS [9], NT-proBNP in ACS [10], and myocardial injury post-PCI).

In a survey on patients with an atherothrombotic event (myocardial infarction or ischemic stroke), the rate of a first recurrent event at 1 year was almost double for patients with multisite atherosclerotic disease [11] as opposed to those with a single disease location (5.6\% vs. $2.9 \%$ ) [12]. Moreover, a recent study performed in the acute setting showed that incorporating clinical and paraclinical variables into a single score (namely, CSS) increases the overall ability to predict a broad spectrum of ischemic endpoints. In addition, two studies $[13,14]$ demonstrated an inverse correlation between SS and renal function both in the stable and acute setting of CAD (the higher the SS, the greater the renal dysfunction). We previously showed that atherosclerotic renal artery stenosis (RAS) prevalence in STEMI patients is significant (16\%) and also has a strong connection with eGFR [15]. A cohort of consecutive STEMI patients prospectively investigated per protocol for presence and severity of RAS and renal function offers a unique perspective that would tie the various iterations of the SS/CSS and refine its prognostic capabilities.

Thus, the aims of this analysis were: 1) to stratify the study population according to Myocardial Infarction SYNTAX Score (MI SS) and the Myocardial Infarction Clinical SYNTAX Score (MI CSS), and to identify those variables correlated with the higher score; 2) to evaluate for the first time the predictive power of MI SS/CSS for RAS presence.

\section{Material and methods}

\section{Study population}

This analysis uses data from the REN-ACS study (Cardiovascular, Renal and Metabolic Profile in Patients with Acute Myocardial Infarction Included in the Romanian National Program of Primary Percutaneous Revascularization - a Single Center Observational Study), which included 181 consecutive patients enrolled between October 2014 and March 2015 (ClinicalTrials.gov registration number NCT02388139). Study design and population, coronary and renal angiographic assessment, body composition analysis and arterial stiffness measurements have been previously described [15]. The primary endpoint was assessment of RAS incidence in consecutive STEMI patients included in the Romanian National Program of Primary Percutaneous Revascularization. The study was approved by the "Gr. T. Popa" Iasi University Ethics Committee. All patients provided written informed consent. No sex-based or racial/ethnic-based differences were present. The investigation of the cardio-renal-metabolic profile in patients with renal atherosclerotic disease was predefined as a secondary outcome.

\section{Inclusion criteria and measurements}

Inclusion criteria were age > 18, confirmed diagnosis of STEMI and enrollment in the Romanian National Program of Primary Percutaneous Revascularization. We followed the European CARDS registration data standards to gather data on medical history, cardiovascular risk factors and Killip class [16]. Medical history consisted of information related to CAD and RAS, such as previous $\mathrm{PCl}$, chronic kidney disease (CKD), stroke, chronic heart failure (CHF), and peripheral artery disease. The biological analysis recorded values for serum glucose, hemoglobin, leucocyte, platelets, total cholesterol, high-density lipoprotein (HDL) and low-density 
lipoprotein (LDL) fractions, uric acid, CRP, Tnl, CKMB fraction, serum urea, and creatinine (eGFR by CKD-Epi formula) and was performed on the same day as the primary $\mathrm{PCl}$ procedure. We performed coronary and renal angiography in order to assess and evaluate coronary/renal artery lesions and percent diameter stenosis. We measured through bioimpedance spectroscopy extracellular (ECW), intracellular (ICW), and total body water (TBW), lean body mass (LBM) and fat tissue mass (FTM). At $24 \mathrm{~h}$ after $\mathrm{PCI}$ we acquired carotid-femoral (cf-PWV) and carotid-radial (cr-PWV) pulse wave velocities and the aortic augmentation index. Supplementary information on the measured characteristics was reported previously [15].

\section{Myocardial Infarction SYNTAX Score (MI SS)}

The SYNTAX Score is an algorithm which calculates coronary atherosclerotic burden and its impact on arterial hemodynamics. A severity score is assigned to each segment of the coronary artery corresponding to the quantity of myocardial muscle at risk. The score calculator and therapeutic indications are available on the SS website (www. syntaxscore.com). Our analysis was performed retrospectively by two independent operators. Every significant inter-observer difference required a recalculation. Initially validated for elective nonACS patients, SS proved to be useful in prognostic assessment after ACS [6]. We computed MI SS from the initial angiography of STEMI.

\section{Myocardial Infarction Clinical SYNTAX Score (MI CSS)}

The SYNTAX Score is computed only from angiographic data and has a lower prognostic ability compared with clinical characteristic-based scores (e.g. Age, Creatinine, and left ventricular Ejection Fraction (ACEF) score [17]). CSS combines both scores (SS multiplied by modified ACEF) and is a better predictor for mortality in patients with complex CAD [18]. Our analysis used the formula $\mathrm{CSS}=\mathrm{SS} \times$ modified ACEF score (age/LVEF +1 for every creatinine clearance $10 \mathrm{ml} / \mathrm{min}$ reduction below $60 \mathrm{ml} / \mathrm{min} / 1.73 \mathrm{~m}^{2}$ ). New evidence showed that in patients with ACS undergoing percutaneous intervention CSS had better predictive accuracy for MACE than SS [19]. Thus, we calculated MI CSS values for each patient with STEMI from our study.

\section{Statistical analysis}

Continuous variables are expressed as mean \pm standard deviation and categorical data as number with percent frequency. All data were stratified according to SS tertiles (Table I) and CSS tertiles (Table II). We performed comparisons between each percentile group (having subsequently three proba- bility values, $p 1, p 2$ and $p 3$ ). Normality of variables distribution was checked with the Shapiro-Wilk test. Independent $t$ test and 1-way ANOVA were performed for continuous variable comparisons. Nominal data were compared using the $\chi^{2}$ test.

Stepwise multivariate linear regression analysis was performed to evaluate independent variables correlated with MI SS or MI CSS. We used C statistics and ROC for MI SS and MI CSS in order to compare the predictive performance of both scores for RAS. Youden index values were computed. Statistical analyses were performed with SPSS 19.0 (SPSS Inc, Chicago, IL) and MedCalc (MedCalc Software bvba, Belgium). A two-tailed $p$-value $<0.05$ was considered significant.

\section{Results}

The REN-ACS study enrolled 181 consecutive STEMI patients, of whom $30(16.6 \%)$ had significant renal artery stenosis ( $>50 \%$, defined as RAS+), while 5 of these had bilateral RAS. No significant difference between left and right prevalence of the RA stenosis was recorded. The MI SS ranged from 1 to 46.5 , with a mean \pm SD of 16.95 \pm 8.45 and a median of 17.5 . The MI CSS ranged from 1.22 to 255.15 , with a mean \pm SD of 39.3 \pm 41.3 and a median of 28 . The study group was divided according to MI SS into tertiles as follows: tertile 1 MI SS $\leq 11(n=59)$, tertile 2 MI SS $11.1-$ $19.9(n=61)$, tertile 3 MI SS $\geq 20(n=61)$. In a second approach, the study group was split according to MI CSS into tertiles as follows: tertile $1 \mathrm{MI}$ CSS $\leq 19.2(n=60)$, tertile 2 MI CSS 19.3-38.8 $(n=61)$, tertile 3 MI CSS $\geq 38.9(n=60)$.

\section{Stratification according to SYNTAX Score}

Baseline clinical and paraclinical characteristics together with post-procedural PWV and body composition monitoring (BCM) measurements were divided according to MI SS tertiles and presented in Table I. In the tertiles 2 and 3 subgroups there were significantly older patients with higher prevalence of previous $\mathrm{CAD}$, previous $\mathrm{PCl}, \mathrm{CHF}$, and hypertension. They also had more RAS+ and bi/tri coronary lesions, higher fibrinogen and CRUSADE score, and lower left ventricular ejection fraction (LVEF) and eGFR $(p<0.05)$. No differences were noted for PWV and BCM variables between MI SS tertiles. A stepwise multivariate linear regression including all the variables associated with MI SS was performed. Independent variables correlated with MI SS were LVEF < 40\%, RAS+, history of CHF, and multivascular CAD (Table III).

\section{Stratification according to Clinical SYNTAX Score}

We performed the same stratification in tertiles for the MI CSS (Table II). We excluded from 
Table I. Patients' characteristics according to MI Syntax Score tertiles

\begin{tabular}{|c|c|c|c|c|c|c|}
\hline Variable & $\begin{array}{l}\text { Tertile } 1 \\
\text { MI SS } \leq 11 \\
(n=59)\end{array}$ & $\begin{array}{c}\text { Tertile } 2 \\
\text { MI SS } 11.1-19.9 \\
(n=61)\end{array}$ & $\begin{array}{c}\text { Tertile } 3 \\
\text { MI SS } \geq 20 \\
(n=61)\end{array}$ & $\begin{array}{c}p 1 \\
(I-I I)\end{array}$ & $\begin{array}{c}p 2 \\
(I I-I I I)\end{array}$ & $\begin{array}{c}p 3 \\
(I-I I I)\end{array}$ \\
\hline RAS+, $n(\%)$ & $5(8.5)$ & $8(13.1)$ & $17(27.8)$ & 0.41 & 0.043 & 0.006 \\
\hline Male, $n(\%)$ & $44(74.5)$ & $50(82)$ & $41(67.2)$ & 0.32 & 0.06 & 0.37 \\
\hline Age [years] ${ }^{\star}$ & $59.27 \pm 12.22$ & $61.80 \pm 11.47$ & $63.49 \pm 11.58$ & 0.24 & 0.42 & 0.05 \\
\hline 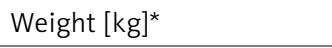 & $84.83 \pm 13.23$ & $85.93 \pm 17.85$ & $80.64 \pm 14.92$ & 0.70 & 0.07 & 0.10 \\
\hline BMI $\left[\mathrm{kg} / \mathrm{m}^{2}\right]^{*}$ & $29.26 \pm 4.36$ & $29.32 \pm 5.09$ & $28.42 \pm 4.53$ & 0.94 & 0.30 & 0.30 \\
\hline History of CAD, $n(\%)$ & $12(20.3)$ & $15(24.6)$ & $28(46)$ & 0.57 & 0.01 & 0.002 \\
\hline History of CKD, $n(\%)$ & $2(3.4)$ & $8(13.11)$ & $3(5)$ & 0.053 & 0.11 & 0.67 \\
\hline History of $\mathrm{PCI}, n(\%)$ & 0 & 0 & $5(8.2)$ & 0.98 & 0.02 & 0.02 \\
\hline History of CHF, $n(\%)$ & $6(10.15)$ & $10(16.4)$ & $20(32.8)$ & 0.31 & 0.035 & 0.002 \\
\hline History of stroke, $n(\%)$ & $6(10.15)$ & $2(3.3)$ & $3(5)$ & 0.13 & 0.67 & 0.27 \\
\hline History of PAD, $n(\%)$ & $3(5)$ & $4(6.5)$ & $4(6.5)$ & 0.73 & 1 & 0.73 \\
\hline History of DM, $n(\%)$ & $9(15.25)$ & $12(19.67)$ & $16(26.2)$ & 0.52 & 0.38 & 0.13 \\
\hline Smoking, $n(\%)$ & $38(64.4)$ & $37(60.65)$ & $38(62.3)$ & 0.98 & 0.98 & 0.98 \\
\hline History of HT, $n$ (\%) & $25(42.3)$ & $35(57.4)$ & $37(60.65)$ & 0.10 & 0.71 & 0.045 \\
\hline Bi\&Tri coro, $n(\%)$ & $15(25.4)$ & $40(65.5)$ & $47(77)$ & 0.001 & 0.16 & 0.001 \\
\hline $\mathrm{Hb}[\mathrm{g} / \mathrm{l}]^{*}$ & $14.25 \pm 1.79$ & $14.91 \pm 4.13$ & $13.82 \pm 1.77$ & 0.26 & 0.06 & 0.19 \\
\hline White blood cells, $n \times 10^{3}$ & $12.007 \pm 4.12$ & $11.899 \pm 4.35$ & $12.170 \pm 3.60$ & 0.89 & 0.71 & 0.81 \\
\hline $\mathrm{PLT}, n \times 10^{3}$ & $240686 \pm 51141$ & $237786 \pm 53449$ & $239122 \pm 72112$ & 0.76 & 0.90 & 0.89 \\
\hline Glu [mg/dl] ${ }^{*}$ & $119.66 \pm 40.73$ & $131.00 \pm 52.71$ & $133.61 \pm 75.24$ & 0.19 & 0.82 & 0.20 \\
\hline Cholesterol total $^{*}$ & $199.15 \pm 53.60$ & $187.73 \pm 44.57$ & $191.27 \pm 44.90$ & 0.20 & 0.66 & 0.38 \\
\hline $\operatorname{LDL}^{*}$ & $115.09 \pm 46.79$ & $107.91 \pm 30.37$ & $114.13 \pm 41.76$ & 0.31 & 0.34 & 0.90 \\
\hline $\mathrm{HDL}^{*}$ & $57.73 \pm 25.23$ & $52.17 \pm 22.13$ & $51.51 \pm 18.31$ & 0.20 & 0.85 & 0.12 \\
\hline CK-MB peak* & $218.37 \pm 201.42$ & $221.46 \pm 183.82$ & $264.12 \pm 273.33$ & 0.93 & 0.31 & 0.30 \\
\hline CK-MB admission* & $79.86 \pm 70.12$ & $75.61 \pm 81.30$ & $93.89 \pm 127.69$ & 0.76 & 0.34 & 0.45 \\
\hline $\mathrm{Fbg}[\mathrm{mg}]^{*}$ & $486.13 \pm 172.45$ & $485.51 \pm 125.70$ & $538.89 \pm 163.28$ & 0.98 & 0.045 & 0.05 \\
\hline CRUSADE $^{*}$ & $23.27 \pm 12.06$ & $25.16 \pm 9.73$ & $29.18 \pm 12.42$ & 0.34 & 0.049 & 0.009 \\
\hline eGFR $^{*}$ & $85.10 \pm 19.27$ & $78.04 \pm 17.97$ & $75.48 \pm 21.76$ & 0.04 & 0.48 & 0.012 \\
\hline BUN : Crea* & $20.66 \pm 9.73$ & $18.04 \pm 5.49$ & $18.84 \pm 6.47$ & 0.07 & 0.45 & 0.22 \\
\hline Alx $x^{*}$ & $23.25 \pm 12.57$ & $23.86 \pm 11.34$ & $21.25 \pm 14.12$ & 0.77 & 0.26 & 0.41 \\
\hline cf-PWV* & $9.14 \pm 1.97$ & $9.35 \pm 2.62$ & $9.65 \pm 2.92$ & 0.61 & 0.55 & 0.26 \\
\hline $\mathrm{cr}-\mathrm{PWV}{ }^{*}$ & $7.25 \pm 1.13$ & $6.84 \pm 1.15$ & $6.92 \pm 1.12$ & 0.051 & 0.68 & 0.11 \\
\hline LVEF < $40 \%, n(\%)$ & $22(37.3)$ & $31(50.8)$ & $34(55.7)$ & 0.13 & 0.58 & 0.042 \\
\hline AFO $[1]^{*}$ & $-1.87 \pm 1.94$ & $-1.85 \pm 2.88$ & $-1.37 \pm 2.62$ & 0.97 & 0.34 & 0.24 \\
\hline RFO (\%)* & $-11.12 \pm 11.56$ & $-10.96 \pm 16.86$ & $-9.77 \pm 16.48$ & 0.95 & 0.69 & 0.60 \\
\hline TBW $[\mathrm{I}]^{*}$ & $40.57 \pm 6.70$ & $40.54 \pm 8.87$ & $38.20 \pm 7.41$ & 0.98 & 0.11 & 0.07 \\
\hline ECW [I] $]^{*}$ & $17.31 \pm 2.21$ & $17.33 \pm 3.02$ & $16.68 \pm 3.33$ & 0.96 & 0.25 & 0.22 \\
\hline ICW [I] ${ }^{*}$ & $23.25 \pm 5.25$ & $23.20 \pm 6.73$ & $21.53 \pm 5.00$ & 0.96 & 0.12 & 0.06 \\
\hline LTM [kg] & $47.51 \pm 14.54$ & $47.48 \pm 17.63$ & $43.55 \pm 13.01$ & 0.99 & 0.16 & 0.19 \\
\hline FTM $[\mathrm{kg}]^{*}$ & $28.96 \pm 12.33$ & $28.73 \pm 13.87$ & $28.50 \pm 10.96$ & 0.92 & 0.23 & 0.82 \\
\hline
\end{tabular}

${ }^{*}$ Mean \pm standard deviation. Bold values are statistically significant. RAS - renal artery stenosis, CAD - coronary artery disease, $C K D$ - chronic kidney disease, $P C I$ - percutaneous coronary intervention, CHF - congestive heart failure, PAD - peripheral artery disease, $\mathrm{Hb}$ - hemoglobin, $\mathrm{LDL}$ - low-density lipoprotein, HDL - high-density lipoprotein, Crea - creatinine, eGFR-estimated glomerular filtration rate, LVEF echo - left ventricle ejection fraction, Aix - augmentation index, cf- and cr-PWV - carotid-femoral and carotid-radial pulsed wave velocity, BCM - body composition monitoring, AFO - absolute fluid overload, RFO - relative fluid overload, TBW - total body water, ECW - extracellular water, ICW - intracellular water, LTM - lean tissue mass, FTM - fat tissue mass. 
Table II. Patients' characteristics according to Clinical Syntax Score tertiles

\begin{tabular}{|c|c|c|c|c|c|c|}
\hline Variable & $\begin{array}{l}\text { Tertile } 1 \\
\text { MI CSS } \leq 19.2 \\
(n=60)\end{array}$ & $\begin{array}{c}\text { Tertile } 2 \\
\text { MI CSS } 19.3-38.8 \\
(n=61)\end{array}$ & $\begin{array}{c}\text { Tertile } 3 \\
\text { MI CSS } \geq 38.9 \\
(n=60)\end{array}$ & $\begin{array}{c}p 1 \\
(I-I I)\end{array}$ & $\begin{array}{c}p 2 \\
(I I-I I I)\end{array}$ & $\begin{array}{c}p 3 \\
(I-I I I)\end{array}$ \\
\hline RAS+, $n$ (\%) & $5(8.33)$ & $6(9.8)$ & $19(31.66)$ & 0.77 & 0.003 & 0.001 \\
\hline Male, $n(\%)$ & $49(81.6)$ & $47(77)$ & $39(65)$ & 0.53 & 0.14 & 0.038 \\
\hline Age [years] ${ }^{*}$ & $54.33 \pm 11.35$ & $62.93 \pm 10.52$ & $67.35 \pm 9.78$ & 0.0001 & 0.018 & 0.0001 \\
\hline Weight $[\mathrm{kg}]^{*}$ & $86.85 \pm 13.26$ & $82.72 \pm 16.79$ & $81.82 \pm 16.16$ & 0.13 & 0.76 & 0.065 \\
\hline BMI $\left[\mathrm{kg} / \mathrm{m}^{2}\right]^{*}$ & $29.49 \pm 4.2$ & $28.24 \pm 4.6$ & $29.27 \pm 5.05$ & 0.12 & 0.24 & 0.80 \\
\hline History of CAD, $n(\%)$ & $10(16.66)$ & $16(26.2)$ & $29(48.33)$ & 0.20 & 0.011 & 0.011 \\
\hline History of CKD, $n(\%)$ & $3(5)$ & $2(3.3)$ & $8(13.33)$ & 0.63 & 0.054 & 0.11 \\
\hline History of $\mathrm{PCl}, n(\%)$ & $1(1.66)$ & 0 & $5(8.33)$ & 0.98 & 0.021 & 0.08 \\
\hline History of CHF, $n(\%)$ & $5(8.33)$ & $11(18)$ & $20(33.3)$ & 0.11 & 0.053 & 0.053 \\
\hline History of stroke, $n(\%)$ & $2(3.33)$ & $4(6.5)$ & $5(8.33)$ & 0.41 & 0.70 & 0.24 \\
\hline History of PAD, $n(\%)$ & $3(5)$ & $3(5)$ & $5(8.33)$ & 0.98 & 0.44 & 0.44 \\
\hline History of DM, $n(\%)$ & $11(18.33)$ & $9(14.75)$ & $17(28.33)$ & 0.52 & 0.06 & 0.19 \\
\hline Smoking, $n(\%)$ & $40(66.6)$ & $37(60.65)$ & $36(60)$ & 0.42 & 0.89 & 0.41 \\
\hline History of HT, $n(\%)$ & $24(40)$ & $37(60.65)$ & $36(60)$ & 0.02 & 0.97 & 0.02 \\
\hline Bi\&Tri coro, $n(\%)$ & $25(41.6)$ & $30(49.2)$ & $47(78.33)$ & 0.40 & 0.001 & 0.001 \\
\hline $\mathrm{Hb}[\mathrm{g} / \mathrm{l}]^{*}$ & $14.90 \pm 4.13$ & $14.46 \pm 1.47$ & $13.62 \pm 2.05$ & 0.43 & 0.011 & 0.033 \\
\hline White blood cells $\left[n \times 10^{3}\right]$ & $11897 \pm 3703$ & $11812 \pm 4256$ & $12371 \pm 4118$ & 0.91 & 0.46 & 0.50 \\
\hline PLT $\left[n \times 10^{3}\right]$ & $241108 \pm 48387$ & $245852 \pm 55358$ & $230475 \pm 72002$ & 0.61 & 0.19 & 0.34 \\
\hline Glu [mg/dl]* & $121.17 \pm 48.53$ & $125.31 \pm 48.66$ & $138.12 \pm 73.50$ & 0.64 & 0.26 & 0.13 \\
\hline Cholesterol total ${ }^{*}$ & $206.34 \pm 51.25$ & $192.19 \pm 41.20$ & $179.41 \pm 47.34$ & 0.09 & 0.11 & 0.003 \\
\hline $\mathrm{LDL}^{*}$ & $122.04 \pm 45.75$ & $110.27 \pm 36.76$ & $104.76 \pm 35.63$ & 0.12 & 0.40 & 0.023 \\
\hline $\mathrm{HDL}^{*}$ & $55.05 \pm 20.94$ & $55.79 \pm 27.57$ & $50.40 \pm 16.11$ & 0.86 & 0.19 & 0.17 \\
\hline CK-MB peak* & $212.80 \pm 180.65$ & $230.37 \pm 204.32$ & $261.40 \pm 274.30$ & 0.61 & 0.48 & 0.25 \\
\hline CK-MB admission* & $72.65 \pm 75.13$ & $83.84 \pm 82.31$ & $92.96 \pm 124.82$ & 0.43 & 0.63 & 0.28 \\
\hline $\mathrm{Fbg}[\mathrm{mg}]^{*}$ & $441.65 \pm 126.65$ & $490.10 \pm 127.19$ & $579.60 \pm 178.58$ & 0.038 & 0.002 & 0.001 \\
\hline CRUSADE* $^{*}$ & $19.93 \pm 9.55$ & $24.39 \pm 9.82$ & $33.40 \pm 11.39$ & 0.013 & 0.001 & 0.001 \\
\hline eGFR* & $91.80 \pm 12.82$ & $81.08 \pm 14.45$ & $65.53 \pm 22.21$ & 0.001 & 0.001 & 0.001 \\
\hline BUN : Crea* & $19.08 \pm 9.54$ & $19.27 \pm 5.14$ & $19.15 \pm 7.21$ & 0.89 & 0.91 & 0.96 \\
\hline Alx $x^{*}$ & $23.09 \pm 12.14$ & $20.43 \pm 13.81$ & $24.87 \pm 11.88$ & 0.26 & 0.06 & 0.41 \\
\hline cf-PWV* & $8.64 \pm 1.66$ & $9.31 \pm 2.39$ & $10.20 \pm 3.12$ & 0.075 & 0.08 & 0.001 \\
\hline $\mathrm{Cr}-\mathrm{PWV}^{*}$ & $7.02 \pm 1.11$ & $6.94 \pm 1.29$ & $7.05 \pm 1.03$ & 0.69 & 0.61 & 0.94 \\
\hline LVEF < $40 \%, n(\%)$ & $9(15)$ & $31(50.8)$ & $46(76.6)$ & 0.001 & 0.003 & 0.001 \\
\hline AFO []$^{*}$ & $1.76 \pm 1.55$ & $-2.41 \pm 3.00$ & -0.90 & 0.13 & 0.004 & 0.029 \\
\hline RFO $(\%)^{*}$ & $-10.46 \pm 9.82$ & $-14.70 \pm 18.44$ & $-6.61 \pm 14.91$ & 0.11 & 0.009 & 0.09 \\
\hline TBW $[\mathrm{I}]^{*}$ & $41.53 \pm 6.81$ & $40.81 \pm 8.30$ & $36.93 \pm 7.42$ & 0.61 & 0.008 & 0.001 \\
\hline $\mathrm{ECW}[\mathrm{l}]^{*}$ & $17.75 \pm 2.62$ & $16.96 \pm 2.68$ & $16.61 \pm 3.27$ & 0.10 & 0.51 & 0.038 \\
\hline ICW [l] ${ }^{*}$ & $23.77 \pm 4.70$ & $23.84 \pm 6.70$ & $20.32 \pm 4.95$ & 0.95 & 0.001 & 0.001 \\
\hline${\text { LTM }[k g]^{*}}^{*}$ & $48.40 \pm 12.50$ & $49.65 \pm 17.95$ & $40.39 \pm 13.08$ & 0.65 & 0.002 & 0.001 \\
\hline FTM [kg] ${ }^{*}$ & $30.36 \pm 10.77$ & $26.15 \pm 14.26$ & $29.73 \pm 11.59$ & 0.07 & 0.13 & 0.76 \\
\hline
\end{tabular}

${ }^{\star}$ Mean \pm standard deviation. Bold values are statistically significant. RAS - renal artery stenosis, CAD - coronary artery disease, $C K D$ - chronic kidney disease, $P C I$ - percutaneous coronary intervention, CHF - heart failure, PAD - peripheral artery disease, $\mathrm{Hb}$ - hemoglobin, LDL - low density lipoprotein, HDL - high density lipoprotein, Crea - creatinine, eGFR - estimated glomerular filtration rate, LVEF echo - left ventricle ejection fraction, Aix - augmentation index, cf-and cr-PWV-carotid-femoral and carotid-radial pulsed wave velocity, BCM - body composition monitoring, AFO - absolute fluid overload, RFO - relative fluid overload, TBW - total body water, ECW - extracellular water, ICW - intracellular water, LTM - lean tissue mass, FTM - fat tissue mass. 
Table III. Multivariate associates of MI SS

\begin{tabular}{|lccc|}
\hline Variable & B value & $95 \% \mathrm{Cl}$ & $P$-value \\
\hline $\begin{array}{l}\text { Renal artery } \\
\text { stenosis }>50 \%\end{array}$ & 3.31 & $0.40-6.23$ & 0.026 \\
\hline Previous CHF & 3.53 & $0.80-6.27$ & 0.012 \\
\hline LVEF < 40\% & 3.33 & $1.2-5.46$ & 0.002 \\
\hline $\begin{array}{l}\text { Number of } \\
\text { affected vessels }\end{array}$ & 6.21 & $3.99-8.42$ & 0.001 \\
\hline
\end{tabular}

the analysis age, LVEF, and eGFR because these were already included in the clinical score formula. In tertiles 2 and 3 there were significantly more females and hypertensives, with higher prevalence of RAS+ and multivascular CAD. Higher tertiles of MI CSS had lower Hgb and total cholesterol, and higher fibrinogen values. Also, in tertiles 2 and 3 we observed significantly higher cf-PWV, and lower absolute fluid overload (AFO), relative fluid overload (RFO), TBW, ICW, ECW, and lean tissue mass (LTM). The independent variables correlated with MI CSS in multivariate linear stepwise regression were: RAS+, cf-PWV, history of CAD, multivascular coronary disease, total cholesterol, and TBW (through ICW, but not ECW) (Table IV).

\section{Clinical SYNTAX Score versus MI SYNTAX Score as RAS+ predictor}

We performed ROC curves and AUCS for MI SS and MI CSS to evaluate their performance and predictive accuracy for RAS+. Both curves are present-

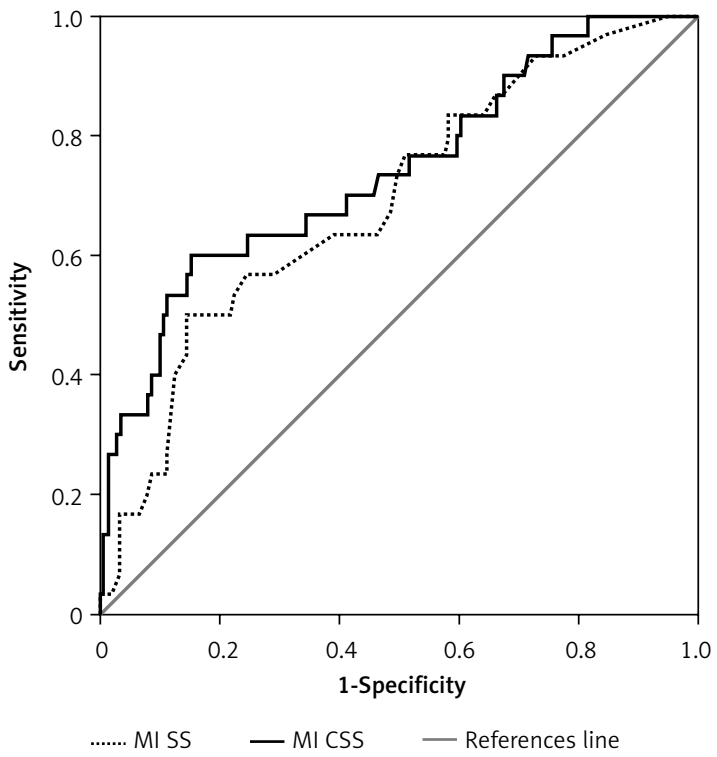

AUC - area under curve of ROC, ROC - receiver operating characteristics. Diaginal segments are produced by ties.

Figure 1. AUC ROC comparison between MI SS and MI CSS
Table IV. Multivariate associates of MI CSS

\begin{tabular}{|lccc|}
\hline Variable & B value & $95 \% \mathrm{Cl}$ & $P$-value \\
\hline $\begin{array}{l}\text { Renal artery } \\
\text { stenosis }>50 \%\end{array}$ & 26.46 & $12.43-40.48$ & 0.001 \\
\hline Previous CAD & 17.69 & $6.48-28.90$ & 0.002 \\
\hline $\begin{array}{l}\text { Number of } \\
\text { affected vessels }\end{array}$ & 16.75 & $6.45-27.04$ & 0.002 \\
\hline Cf-PWV & 3.18 & $1.14-5.21$ & 0.002 \\
\hline TBW & -0.81 & $(-1.46)-(-0.153)$ & 0.016 \\
\hline Total cholesterol & -0.12 & $(-0.22)-(-0.018)$ & 0.022 \\
\hline
\end{tabular}

ed in Figure 1. The AUC for MI SS was $0.69(95 \% \mathrm{Cl}$ : $0.58-0.79)$ and for MI CSS 0.74 (95\% Cl: 0.63-0.84) ( $p<0.05$ for both analyses, DeLong et al.). Comparison of both AUCs yielded no significant differences in predicting RAS+ between the two scores $(p=0.12)$. Youden's Index for MI CSS was 0.45 and for MI SS was 0.35 (Table V) with the same specificity $(85 \%)$ but a higher sensitivity $(60 \%$ versus $50 \%$ ) for MI CSS. The positive likelihood ratio for MI SS is 3.33 and for MI CSS 4. Negative likelihood ratios were 0.58 and 0.47 , respectively. MI CSS has a strong negative predictive value (92\%) but a fair positive predictive value (43\%).

\section{Discussion}

To our knowledge this is the first investigation that uses and compares directly SS and Clinical SS in consecutive STEMI primary PCl settings. Most importantly, the two scores were analyzed and compared for the first time as predictors for RAS+ (until now, both were used exclusively as prognostic factors). Another novelty is that arterial rigidity (PWV) and bioimpedance (BCM) parameters were investigated in relation to $\mathrm{MI} \mathrm{SS/MI} \mathrm{CSS} \mathrm{in} \mathrm{this}$ cohort.

Although the association between CAD severity and peripheral determinations is well known, multisite atherosclerotic disease represents a twofold challenge, in terms of the correct evaluation of prognosis and adequate and personalized management [11].

Previous studies showed a strong relationship between the extent of CAD and RAS presence in elective consecutive patients; namely, the number

Table V. Youden index values

\begin{tabular}{|lcc|}
\hline Variable & MI SS & MI CSS \\
\hline Youden index J & 0.3543 & 0.4477 \\
\hline Associated criterion & $>23$ & $>48.45$ \\
\hline Sensitivity & 50.00 & 60.00 \\
\hline Specificity & 85.43 & 84.77 \\
\hline
\end{tabular}


of coronary arteries involved raises RAS prevalence [20]. Moreover, there was reported an almost two times higher risk of recurrence at 1 year for patients with previous history of an atherothrombotic event and multisite disease (vs. single disease location) [12]. Furthermore, a systematic review published in 2009 described a significant increase of RAS prevalence with the number of coronary arteries involved (5.5\% for 1 -vessel, $9.7 \%$ for 2 -vessel and $15.1 \%$ for 3 -vessel disease, $p<0.0001$ ) [21].

Also, we recently reported first that multivascular CAD is an independent predictor for RAS in STEMI patients [15]. The highest RAS prevalence (54\%) was identified in a group of patients with CHF: 1 of 2 patients with LVEF $<40 \%$ and clinical symptoms and signs of CHF proved to have RAS+ [22].

Multivariate regression analysis showed a strong correlation between MI SS and various parameters that characterize extensive multisite atherosclerosis (bi/three vessel CAD, LVEF $<40 \%$, clinical CHF, and RAS+). Thus, it is safe to assert that patients with RAS+ have a high SS score, or, in other words, a high SS score is correlated with multisite atherosclerosis. This information - relevant to the risk prediction profile [23] - is immediately available for the prevention of early deterioration of renal function (most importantly use of ACE inhibitors with diuretics or in underhydrated patients). In addition, it is essential to better characterize resistant hypertension cases in the context of unstable CAD. It has already been reported that performing renal angioplasty in the same procedure with primary $\mathrm{PCl}$ could yield favorable outcomes [24].

Our analysis also showed significant correlations between MI CSS and extensive atherosclerosis (RAS+, history of CAD and multivascular CAD), higher arterial rigidity (cf-PWV), and dehydration (lower TBW through ICW, but not ECW) and lower total cholesterol. This interesting observation suggests for the first time that a higher MI CSS is associated with presence of RAS+, higher vascular rigidity and dehydration.

We previously reported a high level of dehydration in most of our STEMI patients, significantly higher for those with RAS+. The strong correlation between dehydration and a higher MI CSS indicates either that the hydration status could be a novel risk factor in systemic atherosclerosis development, or that dehydration is a consequence of an extended atheromatous systemic disease (promoting water retention, electrolytic imbalance and hormonal changes).

As a specific aim we evaluated the ability of both $\mathrm{MI}$ scores to predict RAS+. Both ROC AUCS showed a comparably good C-statistic (0.69 for MI SS, 0.74 for MI CSS, $p<0.001$ for both). However, the addition of 4 variables (age, LVEF, weight, and serum creatinine) to MI SS increased the pre- diction power of MI CSS for RAS+ (without statistical significance, $p=0.12$ ).

Despite the lack of strength in these data, as a screening test, the negative interpretation of the results (CSS < 44) is very good at reassuring that a patient does not have RAS (negative predictive value $(\mathrm{NPV})=92.0 \%$ ) and at this initial screen correctly identifies $85 \%$ of those who do not have RAS. For example, in patients with increased creatinine levels and a CSS below the 44 cutoff, we can be sure that the elevation of creatinine is not generated by RAS (therefore, angiotensin-converting enzyme inhibitors (ACEi) can be administered in an AMI setting). On the other hand, the weak results in confirming the disorder (positive predictive value (PPV) $=43 \%$ ) show the necessity of further investigations. Therefore, we intend to develop a better predictor incorporating into CSS a novel biomarker $[25,26]$ in order to increase sensitivity from $60 \%$ to $80 \%$, which would support the very good current specificity of $85 \%$.

The low number of total patients in our study group and the low number of RAS+ patients represent a limitation, as well as the possible referral bias, given that the research was performed in a single center. Furthermore, the fair sensitivity of the predictive scores may lead to a limited clinical value of the score assessment. If we had chosen a different threshold for RAS significance, we could have recorded a different relevance for the variables investigated. Moreover, we did not perform a trans-stenotic gradient in order to determine RAS hemodynamic relevance.

In conclusion, we calculated the SYNTAX Score and the Clinical SYNTAX Score in consecutive STEMI patients referred for primary $\mathrm{PCl}$. Both scores correlated with extensive atherosclerotic disease and presence of RAS+. Moreover, MI CSS was associated with higher vascular rigidity and dehydration. This extended score proved to be a good predictor tool for RAS+, with higher specificity and negative predictive value. Identifying RAS through a simple formula improves estimation of MACE risk [27], facilitates the adjustment of antiplatelet, anticoagulant, and angiotensin-converting enzyme inhibitor treatment [28], and helps limit progression to endstage renal disease. Moreover, even if the clinical value of performing renal angioplasty is debatable and not widely accepted, performing this procedure in the same session with primary $\mathrm{PCl}$ could and should be tested in a formal prospective study. This could allow us to assess whether this procedure and its timing could effectively yield favorable outcomes in the setting of AMI patients.

\section{Conflict of interest}

Adrian Covic is a member of the Advisory Board of Fresenius NephroCare. All other authors have no conflicts of interest to declare. 


\section{References}

1. Sianos G, Morel MA, Kappetein AP, et al. The SYNTAX Score: an angiographic tool grading the complexity of coronary artery disease. Eurolntervention 2005; 1: 219-27.

2. Capodanno D, Di Salvo ME, Cincotta G, Miano M, Tamburino C, Tamburino C. Usefulness of the SYNTAX score for predicting clinical outcome after percutaneous coronary intervention of unprotected left main coronary artery disease. Circ Cardiovasc Interv 2009; 2: 302-8.

3. Valgimigli M, Serruys PW, Tsuchida K, et al. Cyphering the complexity of coronary artery disease using the syntax score to predict clinical outcome in patients with three-vessel lumen obstruction undergoing percutaneous coronary intervention. Am J Cardiol 2007; 99: 1072-81.

4. Yadav M, Palmerini T, Caixeta A, et al. Prediction of coronary risk by SYNTAX and derived scores: synergy between percutaneous coronary intervention with taxus and cardiac surgery. J Am Coll Cardiol 2013; 62: 1219-30.

5. Capodanno D, Tamburino C. Integrating the Synergy between percutaneous coronary intervention with Taxus and Cardiac Surgery (SYNTAX) score into practice: use, pitfalls, and new directions. Am Heart J 2011; 161: 462-70.

6. Palmerini T, Genereux P, Caixeta A, et al. Prognostic value of the SYNTAX score in patients with acute coronary syndromes undergoing percutaneous coronary intervention: analysis from the ACUITY (Acute Catheterization and Urgent Intervention Triage StrategY) trial. J Am Coll Cardiol 2011; 57: 2389-97.

7. Ucar H, Gur M, Seker T, et al. Impaired kidney function is associated with SYNTAX score in patients with stable coronary artery disease. Turk Kardiyol Dern Ars 2014; 42: 621-8.

8. Duran M, Uysal OK, Gunebakmaz O, et al. Glomerular filtration rate is associated with burden of coronary ath erosclerosis in patients with acute coronary syndrome. Angiology 2014; 65: 350-6.

9. Karadeniz M, Duran M, Akyel A, et al. High sensitive CRP level is associated with intermediate and high Syntax score in patients with acute coronary syndrome. Int Heart J 2015; 56: 377-80.

10. Kurtul A, Yarlioglues M, Murat SN, et al. N-terminal probrain natriuretic peptide level is associated with severity and complexity of coronary atherosclerosis in patients with acute coronary syndrome. Clin Appl Thromb Hemost 2016; 22: 69-76.

11. Tendera M, Aboyans V, Bartelink ML, et al. ESC Guidelines on the diagnosis and treatment of peripheral artery diseases: document covering atherosclerotic disease of extracranial carotid and vertebral, mesenteric, renal, upper and lower extremity arteries: the Task Force on the Diagnosis and Treatment of Peripheral Artery Diseases of the European Society of Cardiology (ESC). Eur Heart J 2011; 32: 2851-906.

12. Ferrieres J, Cambou JP, Gayet JL, Herrmann MA, Leizorovicz $A$. Prognosis of patients with atherothrombotic disease: a prospective survey in a non-hospital setting. Int J Cardiol 2006; 112: 302-7.

13. Coskun U, Orta Kilickesmez K, Abaci O, et al. The relationship between chronic kidney disease and SYNTAX score. Angiology 2011; 62: 504-8.

14. Yan LQ, Guo LJ, Zhang FC, Gao W. The relationship between kidney function and angiographically-derived SYNTAX score. Can J Cardiol 2011; 27: 768-72.

15. Burlacu A, Siriopol D, Voroneanu L, et al. Atherosclerotic renal artery stenosis prevalence and correlations in acute myocardial infarction patients undergoing primary percutaneous coronary interventions: data from nonrandomized single-center study (REN-ACS) - a single center, prospective, observational study. J Am Heart Assoc 2015; 4: e002379.

16. Flynn MR, Barrett C, Cosio FG, et al. The Cardiology Audit and Registration Data Standards (CARDS), European data standards for clinical cardiology practice. Eur Heart J 2005; 26: 308-13.

17. Ranucci M, Castelvecchio S, Menicanti L, Frigiola A, Pelissero $\mathrm{G}$. Risk of assessing mortality risk in elective cardiac operations: age, creatinine, ejection fraction, and the law of parsimony. Circulation 2009; 119: 3053-61.

18. Garg S, Sarno G, Garcia-Garcia HM, et al. A new tool for the risk stratification of patients with complex coronary artery disease: the Clinical SYNTAX Score. Circ Cardiovasc Interv 2010; 3: 317-26.

19. Palmerini T, Caixeta A, Genereux P, et al. Comparison of clinical and angiographic prognostic risk scores in patients with acute coronary syndromes: analysis from the Acute Catheterization and Urgent Intervention Triage StrategY (ACUITY) trial. Am Heart J 2012; 163: 38391, 91.e1-5.

20. Weber-Mzell D, Kotanko P, Schumacher M, Klein W, Skrabal F. Coronary anatomy predicts presence or absence of renal artery stenosis. A prospective study in patients undergoing cardiac catheterization for suspected coronary artery disease. Eur Heart J 2002; 23: 1684-91.

21. de Mast Q, Beutler JJ. The prevalence of atherosclerotic renal artery stenosis in risk groups: a systematic literature review. J Hypertens 2009; 27: 1333-40.

22. de Silva R, Loh H, Rigby AS, et al. Epidemiology, associated factors, and prognostic outcomes of renal artery stenosis in chronic heart failure assessed by magnetic resonance angiography. Am J Cardiol 2007; 100: 273-9.

23. Banach M, Aronow WS, Serban MC, Rysz J, Voroneanu L, Covic A. Lipids, blood pressure and kidney update 2015. Lipids Health Dis 2015; 14: 167.

24. Su CS, Liu TJ, Tsau CR, et al. The feasibility, safety, and mid-term outcomes of concomitant percutaneous transluminal renal artery stenting in acute coronary syndrome patients at high clinical risk of renal artery stenosis. J Invasive Cardiol 2013; 25: 212-7.

25. Gluba-Brzozka A, Michalska-Kasiczak M, Franczyk B, et al. Markers of increased atherosclerotic risk in patients with chronic kidney disease: a preliminary study. Lipids Health Dis 2016; 15: 22.

26. Gluba-Brzozka A, Michalska-Kasiczak M, Franczyk-Skora B, Nocun M, Banach M, Rysz J. Markers of increased cardiovascular risk in patients with chronic kidney disease. Lipids Health Dis 2014; 13: 135.

27. Franczyk-Skora B, Gluba-Brzozka A, Wranicz JK, Banach $M$, Olszewski R, Rysz J. Sudden cardiac death in CKD patients. Int Urol Nephrol 2015; 47: 971-82.

28. Franczyk-Skora B, Gluba A, Banach M, Rysz J. Treatment of non-ST-elevation myocardial infarction and ST-elevation myocardial infarction in patients with chronic kidney disease. Arch Med Sci 2013; 9: 1019-27. 